\title{
Circuit
}

Musiques contemporaines

\section{Gradus ad Infernum (troisième partie) : entrevue avec Ferdinand Larven Niemantz, " penseur de la musique " et auteur de Musical Compositions of the Century Gradus ad Infernum (Part Three), Interview with Ferdinand Larven Niemantz, "Thinker in Music" and Author of Musical Compositions of the Century}

\section{John Rea}

Volume 26, numéro 1, 2016

John Rea : une masquographie raisonnée

URI : https://id.erudit.org/iderudit/1036061ar

DOI : https://doi.org/10.7202/1036061ar

Aller au sommaire du numéro

Éditeur(s)

Les Presses de l’Université de Montréal

ISSN

1183-1693 (imprimé)

1488-9692 (numérique)

Découvrir la revue

Citer ce document

Rea, J. (2016). Gradus ad Infernum (troisième partie) : entrevue avec Ferdinand Larven Niemantz, « penseur de la musique » et auteur de Musical Compositions of the Century. Circuit, 26(1), 73-85. https://doi.org/10.7202/1036061ar
Résumé de l'article

Dans ce troisième et dernier volet d'une entrevue accordée à John Rea en 1996 par ce " penseur de la musique ", Niemantz révèle des rencontres - tant réelles qu'apparentes - jusqu'ici inconnues entre Helmholtz et Brahms, Brahms et Stravinsky, Mann et Niemantz par le biais d'Adorno, et entre Niemantz et Gérard Grisey. Niemantz se prononce également sur une théorie de la couleur instrumentale. 


\section{Gradus ad Infernum (troisième partie): entrevue avec Ferdinand Larven Niemantz, "penseur de la musique" et auteur de Musical Compositions of the Century}

\section{JOHN REA}

(traduit de l'anglais par Béatrice Rea)

La troisième et dernière entrevue, qui remonte à l'automne 1996, figure ci-dessous.

John Rea: Je suis très heureux que nous ayons enfin l'occasion aujourd'hui de discuter non seulement de vos compositions, surtout vos œuvres pour orchestre, mais aussi de vos projets futurs. Si vous êtes d'accord, nous parlerons des connaissances que votre père [Alwa] vous a transmises alors que vous étiez adolescent [à Vienne], et de vos études musicales et philosophiques aux États-Unis avec votre "second" père, [Theodore Wiesengrund] Adorno.

Pouvons-nous commencer par le commencement, par les leçons de composition paternelles qu'un étudiant de Brahms en fin de carrière vous avait données?

Ferdinand Larven Niemantz: Si seulement je pouvais...

J. R.: (...?) Je repensais à une histoire que vous m'aviez déjà racontée, que votre père vous avait racontée. À 27 ans, Brahms s'installe secrètement à Heidelberg même s'il annonce à ses amis qu'il déménage à Winterthur [Suisse, en 1860], ce qu'il a fini par faire éventuellement.
F. L. $\mathbf{N}$. : Ce n'est pas vers ces sujets que mon esprit se tourne, John, mais plutôt vers le sentiment d'avoir été agressé. Vers mon incapacité à le maîtriser depuis l'entretien d'hier.

J. R. : Oh non! Mais je... suis sincèrement désolé si mes questions vous ont paru déplacées, mais je vous assure...

F. L. N. : Non, non. Pas vous, John. Non. Ces policiers... qui nous ont rendu visite hier soir ont fait de moi - la victime d'une agression - un être honteux, coupable et blessé à nouveau par les actions délétères des autres, une technique alternative à valeur appliquée que je n'avais absolument pas prévue. Je vais très certainement examiner cette question de plus près. Sachez que le fracas du congrès [de la Société des technologies alternatives à valeur appliquée (STAVA)] n'était pas un cas de combustion politique spontanée...

J. R. : Peut-être pourrions-nous retourner à... Je suis soulagé d'entendre cela, Ferdinand. Enfin, je suis désolé d'apprendre que vous vous sentez si...

Bon, retournons donc à cette histoire de Heidelberg et à comment, grâce à cela, des concepts nouveaux et 
importants concernant la texture, l'harmonie et l'espacement des voix vous ont été transmis. Tel que je le comprends, votre père vous a curieusement parlé de Stravinsky dans ce contexte. C'est bien cela?

F. L. N.: Oui, Stravinsky a aussi été victime d'une agression. Absolument, je m'en souviens: la police lui a aussi rendu visite, à Boston rien de moins, à cause de son arrangement [1941] symphonique politiquement piquant du The Star-Spangled Banner. J'avais alors 19 ans, et il avait dirigé l'Orchestre symphonique de Boston le 13 janvier 1944'. Le jour suivant, alors qu'il lui restait encore deux concerts à donner, des détectives lui ont remis une ordonnance de cesser et de s'abstenir d'interpréter son adaptation de l'hymne.

Oh... Mais ne serait-ce pas le jour de votre naissance? Je peux très bien m'imaginer comment il s'est senti. En temps de guerre. Comme vous allez bientôt pouvoir l'apprécier, l'interprétation incendiaire de l'hymne national américain de Jimi [Hendrix] en 1969 est digne d'une mention spéciale dans mon nouveau livre, accompagnée d'une analyse exceptionnelle des couleurs décroisées et séditieuses de la guitare électrique. Ah, le temps de guerre.

J. R. : Merci beaucoup, Ferdinand... de vous rappeler mon anniversaire. Mais je faisais plutôt référence aux leçons à l'Hôtel Excelsior où votre père Alwa vous avait parlé d'une visite secrète à un laboratoire universitaire qu'un Brahms curieux mais troublé avait effectuée, et comment cet événement avait influencé sa musique ainsi que la vôtre?

1. Boston Symphony Orchestra, Centennial Program 20002001, p. 29; voir aussi Eric Walter White (1979), Stravinsky, the Composer and his Works, University of California Press, p. 547.
F. L. N. : Alwa, mon père - oui, bien sûr, merci - m'a raconté deux moments critiques dans la vie de Brahms, deux moments très progressistes, si je puis m'exprimer ainsi. Je crois que ces deux événements doivent être davantage célébrés sans réserve par les musicologues.

Sachez que Brahms est le premier à avoir fait un enregistrement sonore d'une pièce de musique de concert lors des sessions que la compagnie d'Alwa [sic] Edison avait organisées au Grand Hôtel de Vienne en $1889^{2}$. Pour la plupart des commentateurs, cet événement semble excentrique, pour ne pas dire inhabituel, pour la persona que nous supposions être Brahms: ce compositeur tief und dunkel, une image déformée et perpétrée par tant d'historiens de la musique peu judicieux. En vérité, il chérissait Höhen und Licht.

Et c'est durant le deuxième événement, qui s'est en fait déroulé avant [la session d'enregistrement], qu'il a révélé son intérêt secret pour la nature du Klang et pour la reproduction sonore comme telle. Lors de la création en 1885 de sa dernière symphonie dirigée par [Hans von] Bülow à Meiningen, et pour laquelle mon jeune père s'était spécialement déplacé, le bruit a couru que quelques-uns des matériaux thématiques de l'œuvre tiraient leur source d'un incident impliquant l'ouïe de Brahms et d'une désorientation passagère dans ses oreilles subie 25 ans plus tôt lors d'une visite furtive d'un laboratoire de [Hermann von] Helmholtz à l'Université de Heidelberg, mon alma mater.

J. R. : Cela aurait eu lieu à l'été 1860?

2. Voir Jonathan Berger et Charles Nichols (1994), "Brahms at the Piano: An Analysis of Data from the Brahms Cylinder", Leonardo Music Journal, vol. 4, p. 23-30. 
F. L. N.: Oui, parce qu'au début du printemps de la même année, Brahms avait vu son Manifesto contre les "modernistes" [Liszt, Wagner, et al.] tourner au désastre. [Mon père] Alwa avait affirmé que Brahms, humilié, afin de reconstituer ses réserves psychiques, s'était tourné vers la science - comme nous devons tous le faire un jour ou l'autre - pour trouver des réponses factuelles et indéniables, ainsi que les dernières découvertes.

Si ma mémoire est bonne, votre thèse de doctorat aborde des questions similaires concernant la faction lisztienne de ce débat important dans I'histoire de la composition ${ }^{3}$.

J. R. : En effet, ce sujet est discuté dans une partie de ma thèse, merci. Mais vous disiez...

F. L. N.: Père a conjecturé que la rencontre avec Helmholtz n'a pas seulement confirmé le pouvoir extraordinaire du Farbklänge ["couleur des tons"], terme que Brahms a offert à Helmholtz qui a ensuite opté pour Klangfarben ["ton des couleurs, timbre"], mais a aussi fourni une explication plus plausible au soi-disant délai dans la composition de sa première symphonie. Bien sûr, le vieil homme et le jeune adulte s'étaient largement inspirés du Zur Farbenlehre [Théorie des couleurs, 1810] de Goethe, qui en réalité n'était pas du tout une théorie; aucune trace d'experimenta crucis ["expériences cruciales"].

Un an auparavant [1859], Helmholtz avait conçu le prototype d'un appareil qu'il avait appelé le Resonator

3. [ndlr] John Rea (1978), Franz Liszt's "New Path of Composition": The Sonata in B Minor as Paradigm, thèse de doctorat, Université de Princeton. [“résonateur»]. C'était un dispositif ingénieux fait à partir de sphères en verre soufflé de tailles différentes. Ressemblant à une sorte de boule de Noël sur le côté, il avait une ouverture de chaque côté et la plus petite des deux était destinée au meatus acusticus externus, le conduit auditif de l'oreille externe. Grâce à cet outil, Helmholtz a établi les parties constituantes des sons complexes qui pénétraient par l'ouverture la plus grande, puisque la physique du globe intensifiait les fréquences de résonance: il avait découvert les "harmonies de la nature" enfermées au sein des choses vibrantes. Lorsque Brahms a porté les résonateurs à ses oreilles, il n'a pas seulement été stupéfait et désorienté (comme me l'a raconté Père en 1937), mais il a aussi demandé à Helmholtz pourquoi toutes les Farbklänge semblaient émaner d'un seul $\mathrm{H}$ (si bécarre dans le système franco-italien). Le vieux Helmholtz a répondu : " $H$ von $H$ ", faisant référence aux harmoniques provenant du ton H. C'était aussi évidemment une projection de son propre nom sur le mélange sonore dont le démasquage avait été rendu possible grâce à son Resonator, sur lequel il revendiquait alors des droits de propriété intellectuelle. Brahms lui a été éternellement reconnaissant, mais incapable de procurer immédiatement à ses réserves psychiques les bienfaits de cet élan. Je pense que vous voyez, John, comment la composition de ses symphonies s'est vue toujours reportée: cela avait très peu à voir avec le spectre de Beethoven et tout à voir avec le spectre de Helmholtz. Il lui a fallu des années pour atteindre l'hommage audacieux dans sa Quatrième.

J. R.: Une histoire remarquable, Ferdinand. Je suis heureux d'entendre tous ses détails. 
FIGURE 1 Dérivation de Niemantz père de l'accord du Sacre du printemps de Stravinsky.

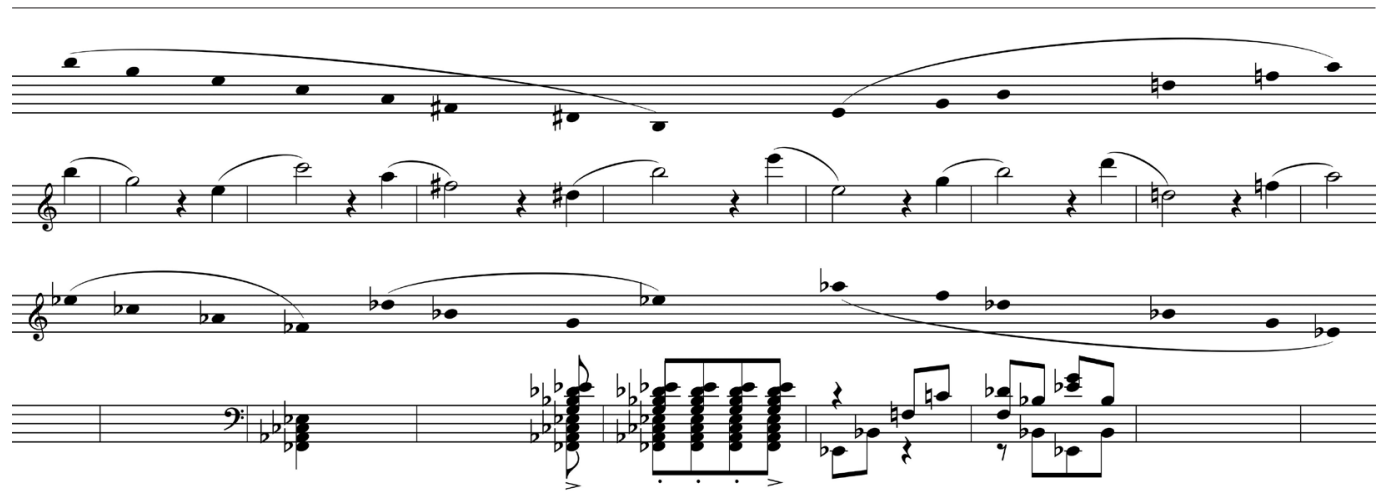

F. L. $\mathbf{N}$ : : Un instant, je vous prie. Vous devez observer par vous-même l'éblouissante preuve de l'hommage établie par Père (de la poche de son veston, il sort une feuille de papier à musique vierge sur laquelle il griffonne rapidement).

Ici [sur la deuxième portée], le thème du premier mouvement de Brahms, et juste au-dessus la façon dont Schoenberg l'a manipulé lors de ces années californiennes passées... à se pencher sur ses Lumpenhörerinnen ["étudiants universitaires grossiers"]. Je n'arrive pas à imaginer qu'ils aient compris quoi que ce soit à son alphabetische Analysierung, que votre professeur à Princeton, [Milton] Babbitt, appelait dans les années 1960 "analyse des classes de tons par intervalle". Et ensuite ici [sur les troisième et quatrième portées], vous pouvez constater l'étonnante manière dont Père resitue le thème.

Mère et moi avons trouvé cette esquisse - le résultat d'une écoute intérieure authentique et profonde - parmi ses affaires personnelles, que nous avions rapidement assemblées à l'[Hôtel] Excelsior avant notre départ précipité pour l'Amérique [en 1938].
Les portées étaient inscrites au dos d'une enveloppe datée de l'été 1913 et pliée méticuleusement en deux sur la barre à la fin de la quatrième mesure. Nous étions à deux doigts de la rater. Mère savait que Père résidait à Paris ce printemps-là.

Je pense que vous pouvez juger comment Père en est venu à représenter exactement les mêmes contours que Schoenberg - mais plusieurs années auparavant, puisque Alwa avait déjà reconnu leur connexion spéciale avec Helmholtz. De gauche à droite, huit tons descendants commencent avec le Höhepunkt $\mathrm{H}$ [si bécarre], compris comme la $16^{\mathrm{e}}$ harmonique, et donc on entend la $13^{\mathrm{e}}$ suivie de la $11^{\mathrm{e}}, 9^{\mathrm{e}}, 7^{\mathrm{e}}, 6^{\mathrm{e}}, 5^{\mathrm{e}}$ et finalement de la $4^{\mathrm{e}}$ harmonique. À droite, la série de six tons ascendants commençant sur la $11^{\mathrm{e}}$ harmonique [mi bécarre] suppose un fondamental une octave plus bas que le premier: un soggetto cavato audacieux sans précédent, " $H$ von $H$ ".

J. R.: Je suis sans mot, Ferdinand! Qu'arrive-t-il sur les troisième et quatrième portées? 
F. L. $\mathbf{N}$.: Père transpose plus bas les huit premiers tons par une quinte augmentée et le deuxième groupe de six, par une quinte diminuée... en inversion!

O diabolus missus est in stagnum ignis ${ }^{4} . .$.

Je lis sur votre visage que vous comprenez maintenant l'anxiété terrible qu'il a ressentie lors de cette découverte. Je présume qu'il a noté de mémoire le contenu de la quatrième portée, tout de suite après la première très animée au Théâtre des Champs-Élysées. Ensuite il a souffert d'un horrible sentiment de trahison et de violation. Quatorze points miraculeux de Höhen und Licht tordus dans l'obscurité sulfureuse...

En effet, son oreille interne avait compris correctement la marche harmonique: le degré napolitain en $f a$ bémol descend vers la tonique provisoire en mi bémol. Cependant, dans le système germanique qu'il aurait employé, Fes [fa bémol] puis Es [mi bémol] résonnent comme - maintenant, écoutez-moi bien dire cela vite - Fes Es - "fesses". J'ai tendance à penser que cette intuition prodigieuse lui a rappelé les quatre cents "coups" de sa jeunesse, comme je vous l'ai mentionné l'autre jour, ou la Lurline et son masque de Cric-Crac.

Dès 1944, quand j'ai finalement trouvé le courage de partager l'intuition de Père avec quelqu'un d'autre, j'ai choisi d'instinct "Père" Wiesengrund. Il est alors devenu livide en repensant aussi à la débâcle autour de l'hymne national plus tôt cette année-là à Boston. D’abord, parce qu'il n'avait pas livré lui-même ce stupéfiant diagnostic et sa dérivation. Et parce qu'il était toujours très offusqué malgré le passage de 30 années

4. Niemantz paraphrase le verset 9 du chapitre 20 de l'Apocalypse de Jean: "et diabolus qui seducebat eos missus est in stagnum ignis et sulphuris ubi..." ("Et le diable, leur séducteur, fut jeté dans l'étang de feu et de soufre..."). et insistait encore et encore: "Pour avoir gain de cause, les Slaves poussent toujours à l'extrême."

Je n'ai aucun doute que le chassé harmonique de Stravinsky trouve sa source dans ses années callipyges passées... pod Chinchilla ${ }^{5}$. Selon moi, et selon mon vrai père [Alwa], ce raisonnement explique bien le pli selon pli illustré par cette enveloppe vide à la raie si parfaite.

J. R.: Oh, seigneur...

F. L. N. : ...oui, et c'est une musica ficta effrayante comme je n'en ai jamais entendue.

J. $\mathbf{R} .:(\ldots)$

F. L. $\mathbf{N} .:(\ldots)$

J. $\mathbf{R}$. : Je me demandais si nous pouvions aborder vos propres compositions pour orchestre (il nous reste si peu de temps pour tant de sujets) et comment la façon spectrale de penser et d'entendre a habité votre conception du son orchestral.

F. L. N.: Mais Beethoven ne m'a pas influencé! Le Geistertrio, nonobstant ${ }^{6} \ldots$

J. R. : Oui, je sais, bien sûr. Mais je... je pensais à Helmholtz et au...

F. L. N.: ...M. Resonator n'avait rien à y voir non plus! C'était plutôt les enseignements hôteliers de Père concernant les sons à hauteur fixe de Webern et leur naïf espacement des voix qui m'ont dirigé vers les quatre cavaliers.

5. "Sous le Chinchilla", en russe. Surnom donné à Diaghilev... mais jamais prononcé devant lui.

6. Trio pour violon, violoncelle et piano, op. $70, n^{\circ} 1$, connu sous le nom de Trio des esprits (1807-1808). 
J. R.: ...les "quatre cavaliers"...?

F. L. N. : ... de l'Apocalypse, dans le Livre de la révélation. C'était la guerre. À ce moment pour moi, chronologiquement jeune mais spirituellement mûr, ils représentaient les quatre éléments les plus singuliers avec lesquels un compositeur modèle des patrons: forme, texture, harmonie et rhétorique. De plus, comme le prônait Père, ils pouvaient tous être harnachés par une traction unifiée, Steigerung ["rehaussement, accroissement"]. II m'a dit que le paradigme mis au monde par Webern et localisé principalement dans son quatuor à cordes américain [op. 28, une commande d'Elizabeth Sprague Coolidge, 1936-1938], pouvait être projeté au sein de tapisseries orchestrales lumineuses - lorsque bien manié.

\section{J. $\mathbf{R}$. : Pourquoi ce quatuor en particulier?}

F. L. N.: Même durant ces brèves années, l'amitié de Père pour le jeune Webern lui était devenue très chère. II l'appelait tendrement "Junior" ou Lehrling [ "apprenti»], et le taquinait avec le sobriquet Anton, von Weberinnen ["Anton, issu de tisserandes"]. Bien que son enseignement ait été de force coupé court, les propos de Père sur Brahms et l'" harmonie de la nature" ont laissé leur empreinte. II m'a fallu beaucoup de temps - compte tenu de tous nos déplacements transatlantiques et puis transcontinentaux, et de notre installation définitive dans un coin plutôt nouveau du Nouveau Monde avant de pouvoir revisiter mes projets orchestraux. Ce que j’ai alors fait avec enthousiasme.

Webern avait montré à Père, plutôt à contrecœur de ce que j'ai compris, les 15 premières mesures environ du premier mouvement de son quatuor, à quoi Père avait répondu impatiemment: "Vieille géométrie!?» Webern avait répliqué: "Nouvelle guématrie - Zwölftontechnik, Bach!"

J. R.: Qu'est-ce que cela voulait dire? L'autre jour, vous l'avez mentionné en parlant du nouveau livre.

F. L. N. : Un autre cas plus informel, mais non moins symbolique, d'alphabetische Analysierung.

J. R. : Eh bien, nous savons que l'opus 28 représente une neuvième tentative avec la nouvelle technique et que, pour ce quatuor de neuf minutes, la série débute avec le tétracorde B.A.C.H., bien que personne ne puisse vraiment l'entendre lorsqu'il est joué.

F. L. N. : Père percevait l'espacement des voix et les registres de Webern comme rien de plus qu'une superstition numérologique, tandis que "Junior" croyait qu'il avait proposé une "harmonie de la nature" - à quoi Père s'opposait: "Non, Bach n'est rien de plus qu'une force de la nature."

J. R.: Désolé, Ferdinand, mais je ne vous suis pas. Les "quatre cavaliers" et le modèle fourni par Webern? Qu'est-ce que je ne vois pas? Je veux dire, qu'est-ce que je ne comprends pas?

F. L. N. : (il reprend le papier à musique et écrit) Voici les sons à hauteur fixe [voir figure 2].

J. R. : Wow, là je comprends mieux. Merci. Je vois aussi le $\mathrm{H}$ grave.

F. L. N. : Grâce à l'analyse de Père, ce balayage de sons sous-développé m’a incité à créer mon propre arc-enciel, sonore en tous points, avec tous les 12 tons et une vraie "harmonie de la nature" basée sur $\mathrm{H}$. Ensuite, je 
FIGURE 2 Analyse de Ferdinand Larven Niemantz du début de l'opus 28 de Webern.

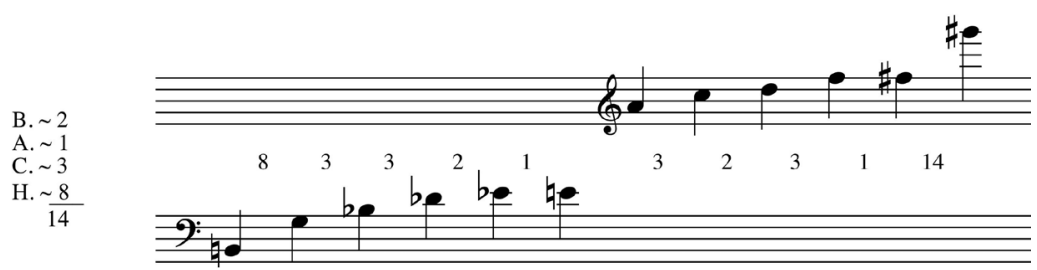

l'ai placé dans une nouvelle composition pour grand orchestre intitulée Les quatre cavaliers, puisqu'elle faisait aussi référence aux quatre octaves où le $\mathrm{H}$ résonnerait au maximum avec tous ses gamins. Ce titre me semblait aussi approprié puisque, peu de temps après notre arrivée à Los Angeles, alors partiellement rurale, javais découvert le Club 4-H, une association nationale pour les jeunes impliqués dans le travail agricole, promouvant les valeurs de tête [head], de cœur [heart], des mains [hands] et de la santé [health].

Les harmoniques 2, 4, 8 et 16, en plus de leur fratrie multicolore: 15 hauteurs simultanées et le son orchestral - tel que je l'imaginais - jaillissant vers l'avant pour une durée totale de 15 minutes. Lumineux et riches, diaphanes et lancinants, chatoyants et frémissants, iridescents et paroxysmaux. L'harmonie et la forme, la texture et la rhétorique, comme autant d'âmes sœurs unies par une étreinte tendue mais aimante, mutuellement récursive... non, mutuellement recombinante...

J. R. : $(\ldots ! ?)$

F. L. N. : Père [Alwa] aurait été très fier de moi - je me répète ces mots encore aujourd'hui. D'un autre côté, "Père" [Adorno] fronçait tout simplement les sourcils lors de nos leçons. "Tu as détissé l'arc-en-ciel", mar- monnait-il en déformant le vers de Keats, qu'il venait de découvrir?

J. R.: Vous sentiez-vous blessé par l'attitu... Je veux dire, par l'aveuglement peu réceptif d’Adorno?

F. L. N.: Par sa surdité, à vrai dire. Si j'avais connu cette référence poétique à l'époque, j'aurais répliqué avec les vers situés trois lignes plus haut: "La philosophie rognera les ailes d'un ange."

J. R. : C'est si triste de penser que votre pièce Les quatre cavaliers n'a jamais été jouée, compte tenu notamment du désastre qui a ensuite frappé vos partitions et effets personnels. Vous m'avez déjà raconté que malgré cette maladie courante dont souffrent tous les jeunes compositeurs - l'envie de performance, comme vous l'avez appelée avec humour -, certaines portions de votre œuvre ont en fait survécu, continuent d'être écoutées, et que tandis...

F. L. N. : . . ouil, au sein de ce fastidieux projet dodécaphonique de Mann, Faustus. Et le fait que ces jeunes ne puissent pas voir leur musique orchestrale innovatrice

7. John Keats, Lamia, $2^{\mathrm{e}}$ partie (1820), lignes $237-238$, "Unweave a rainbow, as it erewhile made / The tender-person'd Lamia melt into a shade". 
sur scène définit bien ce qu'on appelle " musique canadienne". Nous travaillons là-dessus, n'est-ce pas?

Wiesengrund était devenu obsédé par une panoplie de projets - surtout son traité - et irascible au point de subir des poussées d'urticaire répétées, quelque chose d'inouï dans le Los Angeles d'aujourd'hui. Selon moi, il revivait alors une version mise à jour de la querelle des Anciens et des Modernes, opposant maintenant les Horizontaux aux Verticaux. Afin de me réconforter un peu, étant donné sa réaction "paternelle" injustifiée et méprisante envers mes Quatre cavaliers desquels il s'était peu à peu détaché, il m'avait dit que bientôt il insisterait auprès de Mann pour que "Larvenkühn" compose une Apocalypse...

(pause) ...il n'avait même pas remarqué l'incroyable lapsus avec mon nom... Schade.

Et donc, à la fin du roman, ces "glissandos sauvages" - le "rire infernal "-, le "barbarisme" entendus dans l'ekphrasis verbeuse de Mann dans le Lamentatio Doctoris Fausti, expressément là où tous les instruments d'orchestre se retirent en ne laissant qu' un sol aigu au violoncelle, le dernier mot, le son défaillant, qui s'éteint sur un point d'orgue pianissimo. Puis plus rien - le silence, la nuit ${ }^{8}$. Grâce à sa mémoire photographique, Adorno recopie tous ces sons innovateurs de divers passages de ma partition, particulièrement à la toute fin où j'ai manié la $11^{\mathrm{e}}$ harmonique sur un sol aigu. J'ai fait cela avant tout parce que d'ordinaire un violoncelle solo joue faux dans ce registre (avec un sourire diabolique),

8. Niemantz a prononcé ces mots en allemand ("...das hohe g eines Cello, das letzte Wort, der letzte verschwendende Laut, in pianissimo-Fermate langsam vergehend. Dann ist nichts mehr, - Schweigen und Nacht", Thomas Mann [1947], Doktor Faustus, Stockholm, Bermann-Fischer Verlag, p. 745). ce qui nous rapproche bien plus de l'«harmonie de la nature", et ensuite parce que dans le quatuor à cordes de Webern le très aigu Gis gießt ["sol dièse s'écoule"], ce que j'ai refusé de faire. Ce n'est qu'à la fin de mon œuvre que la $16^{\mathrm{e}}$ harmonique descend lentement le long de l'arc-en-ciel afin que le temps s'arrête à la $11^{\mathrm{e}}$ harmonique, à la onzième heure. Puisque les hostilités faisaient encore rage en 1945, c'était mon hommage optimiste et symbolique à l'Armistice de 1919.

J. $\mathbf{R} .:(\ldots)$

F. L. $\mathbf{N} .:(\ldots)$

J. R. : Dans vos propos d'aujourd'hui, je remarque que vous n'avez utilisé les mots "timbre" et "spectre" que très très peu de fois. Par contre, vous semblez beaucoup aimer le terme "arc-en-ciel». Pourquoi?

F. L. $\mathbf{N}$.: La couleur est une condition de lumière blanche. Les artistes plasticiens le comprennent. II en va de même pour la musique: le ton est une condition de son, c'est-à-dire, de bruit. Les compositeurs peinent à le saisir. À mon avis, le timbre évoque quelque chose de similaire à l'étourdissement par percussion, comme pour abattre des animaux. Parce que c'est timbré, n'estce pas?

Mes explorations compositionnelles durant les années 1940 se sont portées sur le retissage et non sur le détissage de l'«harmonie de la nature". Je préférais les effets évanescents d'orchestration produits par une familiarité avec la teinte, un terme qui resplendit de sens pour les chimistes et les chromatographes, mais reste mystérieusement opaque pour les compositeurs et les théoriciens de la musique. La vérité, oui, se rapproche bien plus de la chimie que de la géométrie. (La 
colorimétrie en musique est un sujet bien trop complexe pour l'aborder à cet instant, John. Je ne souhaite certainement pas vous surmener, car vous me semblez fatigué.)

Tout comme avec la sensation visuelle de la teinte le degré auquel un stimulus peut être étiqueté comme similaire à (ou différent) des stimuli qu'on appelle les quatre teintes uniques plus deux (le rouge, le vert, le bleu, le jaune, en plus de l'orange et du violet) j'ai calculé dans ma musique les distances entre les six Farbklängen de base: tuyaux, anches, frites (cuivrées), métaux, bois et membranes (incluant le boyau, aujourd'hui en plastique, et le métal pour les cordophones). Le timbre est primitif, vous pouvez l'entendre. Le reste n'est que bruit.

Dans cette optique, les timbres incomparables de la guitare électrique (mon analyse [de l'hymne de Jimi Hendrix] vous surprendra sincèrement, John) peuvent transcender tous les sons de l'étendue cosmique, à savoir le bruit. Tous les sons connus à ce jour - un vrai "orchestre d'orchestres" - transmettant avec leur...

J. R. : ...merci, Ferdinand, merci! Avant que nous adressions les raisons qui vous ont poussé à changer de carrière, à entamer des études littéraires, je suis très curieux de savoir ce que vous pensez de la propre réorientation de carrière de Stravinsky après la [Seconde] Guerre, le tournant historique, de la perspective de...

F. L. N. : ... d'une technologie alternative à valeur appliquée combinée à la démence et à une quantité non négligeable de charcutage électoral routinier à l’Américaine!

Ce sage [Thomas] von Hartmann et moi nous disputions violemment avec [Robert] Craft - alors un membre jeunot et trop enthousiaste de notre groupe - un peu comme moi-même, d'ailleurs. Nous avons le même âge, vous savez. (J'avoue que notre chère Société a assurément connu son lot de fanatiques kräftige ["effrontés, forts"]...)

Rob [Craft] présumait simplement qu'il serait capable de produire une experimentum crucis. Son défi, nous l'avons tous constaté, était de recourir à... une... "pierre philosophale", qui pour lui incarnait le dodécaphonisme, surtout dans son genus contrapuntisticum. Sautant de joie comme un enfant, il se réjouissait à la pensée révolutionnaire de pouvoir convertir un Moderne en un Ancien.

En y repensant, c'était quasiment un cas de maltraitance de personne âgée.

Personne ne devrait écrire de la musique après l'âge de 70 ans. Écrire des livres, peut-être, oui, mais pas de la musique. C'est une autre raison pour laquelle la musicologie devrait passer à autre chose et explorer la musique populaire en plus de détails, puisque c'est là que les artistes meurent prématurément - comme ils le faisaient autrefois dans le passé glorieux de la musique, à la grande joie et pour le bénéfice historiographique des jeunes chercheurs en musique. Pensez à Purcell, Pergolèse, Mozart, Schubert, Bellini, Lekeu, [Lili] Boulanger, Vivier...

(il rit) Il faudra attendre un autre siècle avant qu'on déchiffre la Magna Carter ${ }^{9}$.

J. R.: $(\ldots ! ?)$

9. Niemantz fait ici référence à Elliot Carter (1908-2012), qui avait alors 88 ans. 
F. L. $\mathbf{N}$. : Le contrepoint est bien plus simple que l'harmonie, car elle est le contrepoint multiplié par quatre, sept ou dix. C'est pourquoi l'harmonie n'est plus enseignée correctement dans les écoles. Et quand elle est enseignée, ce n'est qu'à des fins reliées à son rôle de soutien dans le dévoilement de mélodies embarrassantes. En frappant à la porte d'un compositeur, l'harmonie supplie: "Auriez-vous l'obligeance de me laisser rentrer?" Alors que le contrepoint fait irruption chez les théoriciens, les musicologues et les étudiants sans défense: "Apprenez vos règles, et que ça saute!"

Deux séances par semaine avec Père à l'[Hôtel] Excelsior m'ont appris d'autres leçons vitales. Avant de prendre l'ascenseur pour monter, je devais traverser le lobby majestueux où un orchestre de salon interprétait les airs populaires de l'époque, incluant des arrangements inventifs des classiques. J'ai appris que les instruments monodiques (sauf le piano) chantent des refrains terrestres régis par des règles... comme s'ils rôdaient tous au rez-de-chaussée. Mais les teintes musicales liées, celles que les compositeurs imaginent et construisent pour leurs tableaux sonores, eh bien, elles se trouvent uniquement aux étages supérieurs. Certains sont inoccupés, d'autres densément peuplés. Si vous voulez véritablement composer, espérez que votre hôtel soit un gratte-ciel - pour que vous puissiez vous aussi étriller un arc-en-ciel!

J. R. : Ah? Alors... Pouvons-nous parler brièvement de la catastrophe concernant...?

F. L. N. : ...concernant la publication de la Philosophie der neuen Musik de Wiesengrund?

J. R. : Non, non. Le feu de brousse - votre maison de banlieue, les pertes calamiteuses...
F. L. N.: Précisément! Je me retrouvais au centreville [de Los Angeles] pour mon habituelle leçon de 12 heures chez "Père" [Adorno]. Nos séances débutaient toujours alors qu'il était au téléphone et que moi j’attendais. Souvent, je feuilletais les quotidiens et les magazines éparpillés sur un lutrin du vieux continent. C'était en 1947 - comment pourrais-je l'oublier? Les Américains s'étaient encore convulsivement embarqués dans une campagne électorale présidentielle.

Lorsque son appel s'est terminé, Wiesengrund s'est approché du lutrin: "T'en penses quoi?" J'ai présumé qu'il croyait que j'étais en train d'admirer l'objet, mais spontanément j'ai montré du doigt une photo de [Thomas E.] Dewey [gouverneur de l'État de New York] sur un magazine. En 1948, il était encore une fois le candidat républicain favori désigné pour affronter le président sortant [Harry S.] Truman. J'ai murmuré: "Cette moustache ne vous rappelle-t-elle pas...?" Avant que je puisse terminer ma phrase, le visage d'Adorno est devenu livide. II a chuchoté de façon audible et brusque: "Das stimmt! Am rechten Platz der rechte Mann treu und brav: Schönberg" ["Exactement! $\mathrm{Au}$ bon endroit, la bonne personne, loyale et brave: Schoenberg"]. II voulait que j'entende son jeu de mot d'inversion interlinguistique sur le nom de Truman.

Quelques jours plus tard, il avait pondu un texte sur Stravinsky. Combiné à son essai antérieur sur le "true man": un nouveau volume en dissonance dialectique était prêt à paraître. Durant cette même leçon, la modeste demeure que Mère et moi considérions comme notre chez-soi a été réduite en cendres. C'était tellement typique de ces feux de broussailles frénétiques qui, dans une périphérie autrefois éloignée, étaient déclenchés par des éclairs, sans pluie - ni arc- 
en-ciel. II ne me restait plus que mes notes de cours griffonnées ce jour-là - des annotations à propos du prochain projet de Wiesengrund, en collaboration avec [Hanns] Eisler: un livre sur la composition de musique de film. Mère est décédée peu de temps après.

Par la suite, chaque fois que j'essayais de lire la Philosophie, je devais la redéposer sur-le-champ. Nuit et jour, j'avais des visions hantées d'une campagne politique unilatérale, où "Père" en était le gérant. Puis, dans des cauchemars de célébrations postélectorales, je repérais un "père" angélique jubilant à propos de sa capacité de prédire des résultats en criant: "Tout comme le mercier, le fils du vendeur de chaussures triomphera ${ }^{10}$ !"

J'ai compris à ce moment-là que les choses devaient changer. Il était temps pour moi de me consacrer à nouveau, au propre comme au figuré, à cet ancien concept d'aristos et d'en prendre la responsabilité.

J. R.: Ces événements vous ont poussé à voyager, et finalement vous vous êtes installé dans la région de San Francisco pour étudier à Stanford. Le début des années 1950 a donc été décisif?

F. L. N.: Voyager a changé ma vie, oui. J'avais toujours cru qu'en comparaison avec la vitalité inhérente aux arts, la réalité paraissait dérisoire - puisqu'elle ne peut satisfaire de façon convaincante les exigences rigoureuses de la littérature, de l'art et surtout pas de la musique. Alors pour la première fois depuis 1938, je suis retourné en Europe pour en avoir le cœur net. C'est là-bas que j’ai commencé à saisir à quel point la réalité surpasse en fait les timides tentatives de la dompter.

10. Harry Truman possédait un magasin de vêtements pour hommes, et le père de Schoenberg était vendeur de chaussures.
J'ai réalisé, mais bien plus tard, que la plupart des compositeurs composent en suivant le cours de leur ignorance, alors que moi j'avais acquis une profonde maturité très tôt et que mes plus belles réalisations bien que détruites - avaient déjà été accomplies. Assez tôt dans ma vie, certainement vers la fin de mes études musicales, j'ai compris que... je ne suis personne. Et si je devais devenir quelqu'un, il faudrait que je sois un point de vue, ou un point d'ouie.

J'avais besoin de voyager, car je cherchais à être ébahi. Oui, l'affaire Stravinsky-Craft m’a paru comme la goutte faisant déborder le vase. Et, oui, je n'étais plus intéressé aux miroirs usagés appartenant autrefois à Narcisse. Je voulais quelque chose de bien plus réfléchi. Mon nouveau livre, vous verrez, fournit un indice sur mon état de conscience d'alors.

J. $\mathbf{R} .:(\ldots ! ?)$

F. L. N.: En 1953, par exemple - un peu comme Nietzsche dont la rencontre foudroyante avec [l'opéra] Carmen a changé sa vie -, j’ai découvert purement par accident pendant un voyage en Italie la composition vocale exceptionnelle In un vecchio palco della Scala ${ }^{11}$ dans un spectacle de variétés à Milan. Vous connaissez peut-être la version enregistrée il n'y a pas très longtemps par [Luciano] Pavarotti et Henry Mancini ${ }^{12}$.

La connaissez-vous? Peu importe, vous la trouverez dans ma Catégorie 6) Show-business et démocratisation. Une révélation! Parce qu'ici, la réalité et l'art se

11. Écrite par Pietro Garinei, Sandro Giovannini et Gorni Kramer. Une version interprétée par le Quartetto Cetra en 1953 est disponible ici: <www.youtube.com/ watch $? v=0 F Q k T c f s V u o>$ (consulté le 28 décembre 2015). 12. Mamma (1984), Decca 411 959-2. 
confondent véritablement: tous harnachés par une traction unifiée. L'air microcosmique avait duré à peine cinq minutes et s'était déroulé dramaturgiquement "dans une vieille loge du Teatro alla Scala". Les "spectateurs" y commentent, c'est-à-dire, y chantent somptueusement l'histoire de la forme opératique, de l'édifice même, des nouvelles expressions musicales, des nouvelles expériences (dont la nourriture), et même des Américains à Paris (avec un ingénieux clin d'oreille à Gershwin). Je n'avais jamais rien entendu de tel auparavant.

Voyager me semblait alors tellement plus évident. Je suis allé partout.

J. $\mathbf{R} \cdot:(\ldots ! ?)$

F. L. N.: L'impression que ces forces ont alors produite sur moi est peut-être difficile à concevoir pour vous. C'était, je l'admets, il y a très longtemps. Vous étiez encore enfant, John.

Prenons par exemple un voyage que j'ai fait au printemps passé, que j'adore refaire de temps à autre. Du nord de la Finlande - où je vais pour contempler les arcs-en-ciel nocturnes: l'aurora borealis, ce phénomène luminifère que j’avais espéré pouvoir insérer un jour dans une musique orchestrale ou au moins de chambre (vous et moi savons désormais que cela me sera impossible) - je me rends en Allemagne de l'Ouest pour visiter de bons amis près de Witten. Encore cette année, j’avais accepté une invitation à un concert, selon leur cordialité habituelle, dans le cadre de leur festival de nouvelle musique très animé.

J. R.: Je ne crois pas connaître de festival à Witten.

F. L. N.: Wittener Tage für neue Kammermusik. II existe depuis près de 25 ans maintenant dans sa forme actuelle, mais ses origines datent de 1936. Père [Alwa] en parlait à l'occasion. Eh bien, il faut remonter aux "versets sataniques" de Beethoven pour que l'histoire de la musi...

J. R. : ...pa-pardonnez-moi, Ferdinand! Désolé de vous interrompre, mais... "Versets sa-ta-niques"?

F. L. $\mathbf{N}$. : Oui. Mais je plaisante, John. Je sais très bien que vous détestez voyager.

Opus 120 pour piano: l'ensemble des 33 transsubstantiations d'une Walzer von Diabelli, un effort sans précédent où Beethoven envoie ses auditeurs au paradis... et cette valse pitoyable quoique agréable, ainsi que son soi-disant compositeur, en enfer.

Comme je le disais: il faut remonter à cet acte résolu d'incinération satanique, à ces 33 spécimens granulés vortigineux [sic], pour que l'histoire de la musique soit submergée par une autre œuvre d'art aspirant à la même condition de musique extatique... telle qu'imaginée par der Spagnol ["l'Espagnol", un des premiers surnoms de Beethoven]. Ayant une durée similaire aux Variationen, la création d'une œuvre [Vortex Temporum] de [Gérard] Grisey a eu sur moi un effet brûlant. Qui plus est, c'était un Canadien qui dirigeait les six musiciens allemands ${ }^{13}$ !

Poussière cosmique...

J. R. : ( $\ldots ! ?)$

F. L. $\mathbf{N}$.: Fin de siècle...

J. R. : ( $\ldots ! ?)$

13. La première de Vortex Temporum s'est déroulée le 26 avril 1996 à Witten (Allemagne) sous la direction du chef d'orchestre canadien d'origine trinidadienne, Kwamé Ryan, dirigeant l'Ensemble Recherche (Freiburg im Breisgau). 
F. L. $\mathbf{N}$. : L'estocade... le coup de grâce... l'étourdissement par per-...

J. R.: $(\ldots ! ?)$

F. L. N. : L'arpège-en-ciel de Ravel... pollué...

J. $\mathbf{R} .:(\ldots ! ?)$

F. L. N. : Une apologie du nihilisme considérée comme... John. Je suis désolé. Je me rends compte que vous ignorez cette œuvre, qui m’a laissé - après les semaines passées dans l'harmonieux Cercle Arctique - un arrièregoût flambant...

(en aparté)

this rough magic...

some infernal music...

and deeper than did ever plummet sound

I'll drown my book ${ }^{14} \ldots$

J. $\mathbf{R .}:(\ldots)$

F. L. $\mathbf{N} .:(\ldots)$

J. $\mathbf{R}$. : Nul besoin de vous excuser, Ferdinand. Je connais quelques-unes des œuvres de Grisey et je l'ai rencontré Iorsqu'il est venu à Montréal à la fin des années 1970. Un type très sympathique. J'ai écouté la plupart de ses œuvres spect-... ah,... la plupart de ses compositions depuis. La dernière que j’ai entendue c'était Talea, il y a quelques années.

F. L. N.: Même si j'en suis à l'étape des épreuves en placards, mon éditeur ne m'autorisera pas à ajouter des corrections supplémentaires au livre...

14. Pour des raisons obscures, Niemantz cite la réplique de Prospero dans le dernier acte de The Tempest de Shakespeare (lignes 50, 52 et 56-57). À la ligne 52, il remplace les mots "heavenly music" par "infernal music".
Hmm... Un "type très sympathique", vous dites?

Trop tard pour insérer un paragraphe en Kleintrombe ["tourbillon de poussière", "dust devil" en anglais] à son sujet dans ma Catégorie 9) Calamité, catastrophe et cataclysme.

J. R.: Ah, ahem, je vois qu'il est l'heure de terminer notre entretien si vous ne voulez pas rater votre avion ce soir.

Je vous remercie du fond du cœur, Ferdinand, d'avoir discuté avec moi ces derniers jours. Je veux vous remercier tout particulièrement pour votre franchise, pour la générosité avec laquelle vous m’avez accordé votre temps étant donné les exigences du congrès et d'ailleurs, et pour votre panoplie de sujets si variés.

Certes, nous ne sommes pas allés dans une taverne, comme on avait l'habitude de le faire à Princeton.

\section{F. L. $\mathbf{N}$ : : (il soupire profondément)}

J. $\mathbf{R}$. : Je sais que cela va vous paraître étrange comme demande, mais seriez-vous ouvert à la possibilité de m'accorder une autre entrevue, disons, prochainement? Je sais que votre horaire de voyage et d'enseignement est très chargé. Mais je voyage moi-même un peu plus maintenant, de mon plein gré, et j'aimerais bien avoir l'occasion de vous rencontrer, disons, à Los Angeles. Où que vous soyez en fait.

F. L. $\mathbf{N}$ : : Sehr gerne!

Niemantz a accordé à John Rea deux autres entrevues dix ans plus tard, en 2006, quelques jours avant son $81^{e}$ anniversaire. 